\title{
First-principles study of self-trapped holes and acceptor impurities in $\mathrm{Ga}_{2} \mathrm{O}_{3}$ polymorphs
}

\author{
Tomoya Gake, ${ }^{1}$ Yu Kumagai, ${ }^{2}$ and Fumiyasu $\mathrm{Oba}^{1,3, *}$ \\ ${ }^{1}$ Laboratory for Materials and Structures, Institute of Innovative Research, Tokyo Institute of Technology, Yokohama 226-8503, Japan \\ ${ }^{2}$ Materials Research Center for Element Strategy, Tokyo Institute of Technology, Yokohama 226-8503, Japan \\ ${ }^{3}$ Center for Materials Research by Information Integration, Research and Services Division of Materials Data and Integrated System, \\ National Institute for Materials Science, Tsukuba 305-0047, Japan
}

(Received 22 December 2018; published 9 April 2019)

\begin{abstract}
We investigate the stability of self-trapped holes (STHs) and the acceptor levels of substitutional $\mathrm{Mg}$ and $\mathrm{N}$ impurities in $\alpha-, \beta-, \delta$-, and $\varepsilon-\mathrm{Ga}_{2} \mathrm{O}_{3}$ using first-principles calculations based on the hybrid functional approach to assess their $p$-type dopability. When Fock-exchange and screening parameter values in the Heyd-ScuseriaErnzerhof (HSE) hybrid functional are optimized to satisfy the generalized Koopmans' theorem for a STH level in $\beta-\mathrm{Ga}_{2} \mathrm{O}_{3}$, the band gap is slightly overestimated, while functionals that well reproduce the band gap show slight convex behavior against the fractional electron number. However, the absolute position of the STH level at a fixed geometry is nearly independent of the parameter value, showing that the results are robust as long as the STH geometry and localized electronic nature are appropriately described and the band edges are well reproduced. In all of the polymorphs, holes localize with high self-trapping energies rather than being delocalized. Furthermore, both $\mathrm{Mg}$ and $\mathrm{N}$ impurities introduce polaronic acceptor states, and their acceptor levels lie far above the valence band maximum in all the polymorphs. Thus, the $p$-type doping of the four $\mathrm{Ga}_{2} \mathrm{O}_{3}$ polymorphs seems unfeasible in terms of the STH formation and the related deep, polaronic acceptor nature of the $\mathrm{Mg}$ and $\mathrm{N}$ impurities.
\end{abstract}

DOI: 10.1103/PhysRevMaterials.3.044603

\section{INTRODUCTION}

With a wide band gap of $4.9 \mathrm{eV}$ and $n$-type dopability [1,2], $\beta-\mathrm{Ga}_{2} \mathrm{O}_{3}$ is a promising deep ultraviolet (UV) transparent conductor for applications such as power electronic devices and solar blind UV photodetectors [3-8]. The carrier concentration of $n$-type $\beta-\mathrm{Ga}_{2} \mathrm{O}_{3}$ is easily controllable in the range of $10^{16}-10^{19} \mathrm{~cm}^{-3}$ by Si or Sn doping [2,9-11]. Theoretical suggestions include $\mathrm{H}[12]$ and $\mathrm{Nb}$ [13] impurities, in addition to $\mathrm{Si}$ and $\mathrm{Sn}[12,14]$ as shallow donors. In contrast, previous first-principles studies on the $p$-type dopability of $\beta-\mathrm{Ga}_{2} \mathrm{O}_{3}$ have revealed a lack of shallow acceptors $[15,16]$ and formation of self-trapped holes (STHs) [17-19], both of which hinder efficient $p$-type doping. Apart from exceptions including $\mathrm{Cu}$ (I) and $\mathrm{Sn}$ (II) oxides, the upper valence bands of oxides are mainly composed of $\mathrm{O} 2 p$ orbitals and have small energy dispersions compared with other compounds such as nitrides, phosphides, and arsenides [20-26]. The relatively localized valence states could lead to STH formation and, therefore, low-hole mobility [17]. This is one reason $p$-type doping is challenging for many oxides. In addition, formation of deep acceptor states is closely related to STH stability, as such deep states typically result from polaronic hole trapping $[16,27-30]$. Hence, STH stability is the primary indicator of

\footnotetext{
*oba@msl.titech.ac.jp

Published by the American Physical Society under the terms of the Creative Commons Attribution 4.0 International license. Further distribution of this work must maintain attribution to the author(s) and the published article's title, journal citation, and DOI.
}

$p$-type dopability, particularly for oxides with $\mathrm{O} 2 p$-derived valence bands.

$\mathrm{Ga}_{2} \mathrm{O}_{3}$ has at least five polymorphs with corundum $(\alpha)$, monoclinic $(\beta)$, defective-spinel $(\gamma)$, bixbyite $(\delta)$, and orthorhombic $(\varepsilon)$ structures [31], among which the $\beta$ phase is commonly obtained and assumed to be the most thermodynamically stable under typical growth conditions. A previous first-principles lattice dynamics study showed that the formation free energy increases in the order $\beta, \varepsilon, \alpha, \delta$, and $\gamma$ in a wide temperature range [32]. Experimental and theoretical studies on phases other than the $\beta$ phase are limited. Among the metastable phases, $\alpha-\mathrm{Ga}_{2} \mathrm{O}_{3}$ has been extensively studied [33-35] and its alloy with $\alpha-\mathrm{Al}_{2} \mathrm{O}_{3}$ has attracted attention in band-gap engineering [36,37]. In addition, doping of $\alpha-\mathrm{Ga}_{2} \mathrm{O}_{3}$ with Sn leads to high $n$-type conductivity [38], as in the case of $\beta-\mathrm{Ga}_{2} \mathrm{O}_{3}$ [2,9]. $\varepsilon-\mathrm{Ga}_{2} \mathrm{O}_{3}$ has been suggested as a candidate material for polarization-induced two-dimensional electron gas devices [39]. A previous first-principles study indicates that STHs in the $\varepsilon$ phase are rather stable as in the case of the $\beta$ phase [40]. There is little work on the properties and applications of $\delta$ - and $\gamma-\mathrm{Ga}_{2} \mathrm{O}_{3}$ [41,42]. A detailed study on these $\mathrm{Ga}_{2} \mathrm{O}_{3}$ polymorphs is of interest because atomistic structures dissimilar from that of the $\beta$ phase could cause different behaviors in carrier doping and transport. In particular, characterizing their STHs would be an important step in understanding their $p$-type dopability.

Many first-principles studies have been done on properties of point defects and STHs in semiconductors, including those reported experimentally and predicted theoretically [25,43-51]. Such calculations are typically based on density functional theory (DFT) or hybrid DFT. For the exact density functional, total energy is a piecewise linear function $E(N)$ 
for the fractional electron number $N$ with the derivative discontinuity at the integer $N$ [52]. This is the generalized Koopmans' theorem (gKT). Conventional density functionals such as those based on the local density approximation and generalized gradient approximation (GGA) deviate from this condition. Because of the convex behavior associated with the delocalization error, the derivative discontinuity at the integer $N$ cannot be described correctly, causing underestimation of band gaps and incorrect description of localized states [53,54]. Concave approximations such as Hartree-Fock (HF) exhibit opposite trends and localization error [53,54]. Hybrid functionals inherit convex and concave natures from the local/semilocal density functionals and the HF approximation, respectively, and these errors can be canceled by tuning the amount of each contribution. It has been shown that hybrid functionals improve the description of band gaps and localized states over local/semilocal functionals for a variety of semiconductors and insulators [25,55-61]. Of these, the Heyd-Scuseria-Ernzerhof (HSE) range-separated hybrid functional [62] is probably the most used approximation to reproduce the band gaps of solids with small to medium gaps, but underestimation prevails for wide-gap systems when standard values of Fock-exchange and screening parameters are used [55,63]. For such wide-gap systems including $\beta-\mathrm{Ga}_{2} \mathrm{O}_{3}$, the amount of Fock exchange is often tuned by referring to experimental band gaps [12,13,15-17,64]. By doing so, localized states such as STHs cannot always be well described in terms of the convexity of the functional $[18,65]$. It is therefore important to assess whether the linearity and derivative discontinuity conditions are simultaneously satisfied [18,65-67].

In this paper, we report on the energetics and electronic states of STHs in four $\left(\alpha, \beta, \delta\right.$, and $\varepsilon$ ) $\mathrm{Ga}_{2} \mathrm{O}_{3}$ polymorphs via first-principles calculations based on the hybrid functional approach. First, we investigate the bulk electronic properties of the four $\mathrm{Ga}_{2} \mathrm{O}_{3}$ polymorphs using the HSE hybrid functional with parameter values used for $\beta-\mathrm{Ga}_{2} \mathrm{O}_{3}[12,13,16,17]$. Next, we discuss the Fock-exchange and screening parameters of the HSE functional that satisfy the gKT for a STH level in $\beta-\mathrm{Ga}_{2} \mathrm{O}_{3}$. We then assess the stability of STHs in the four $\mathrm{Ga}_{2} \mathrm{O}_{3}$ polymorphs. Finally, we investigate the acceptor levels of substitutional $\mathrm{Mg}$ and $\mathrm{N}$ impurities in the four polymorphs, and discuss the $p$-type dopability in terms of both STH formation and acceptor doping.

\section{METHODOLOGY}

\section{A. Self-trapping energy and impurity levels}

To discuss the STH stability and acceptor levels of $\mathrm{Mg}$ and $\mathrm{N}$ impurities, we evaluate their formation energies and thermodynamic transition levels. On the basis of the supercell approach, we write the former as

$$
\begin{aligned}
E_{\mathrm{f}}\left[D^{q}\right]= & E\left[D^{q}\right]+E_{\mathrm{corr}}\left[D^{q}\right]-E_{\mathrm{p}} \\
& -\sum_{i} n_{i} \mu_{i}+q\left(\varepsilon_{\mathrm{VBM}}+\Delta \varepsilon_{\mathrm{F}}\right),
\end{aligned}
$$

where $E\left[D^{q}\right]$ and $E_{\mathrm{p}}$ are the total energies of the supercell containing a defect $D$ in charge state $q\left(D^{q}\right)$ and the perfect crystal supercell without the defect, respectively. The correction energy $E_{\text {corr }}\left[D^{q}\right]$ is associated with spurious electrostatic interactions in a finite-sized charged supercell $[68,69]$, $n_{i}$ is the number of added $\left(n_{i}>0\right)$ or removed $\left(n_{i}<0\right)$ $i$-type atoms, $\mu_{i}$ is the chemical potential for an $i$-type atom, $\varepsilon_{\mathrm{VBM}}$ denotes the energy level of the valence band maximum (VBM), and $\Delta \varepsilon_{\mathrm{F}}$ is the Fermi level referenced to $\varepsilon_{\mathrm{VBM}}$.

From Eq. (1), the thermodynamic transition level with respect to the VBM for defect $D$ between charge states $q$ and $q^{\prime}, \varepsilon\left(q / q^{\prime}\right)$, is

$$
\varepsilon\left(q / q^{\prime}\right)=\frac{E_{\mathrm{f}}\left[D^{q}, \Delta \varepsilon_{\mathrm{F}}=0\right]-E_{\mathrm{f}}\left[D^{q^{\prime}}, \Delta \varepsilon_{\mathrm{F}}=0\right]}{q^{\prime}-q},
$$

where $E_{\mathrm{f}}\left[D^{q}, \Delta \varepsilon_{\mathrm{F}}=0\right]$ is the formation energy of $D^{q}$ when the Fermi level is at the VBM. The $\varepsilon(0 /-)$ level corresponds to an acceptor level. For example, the acceptor level of an $\mathrm{Mg}$ impurity on a Ga site $\left(\mathrm{Mg}_{\mathrm{Ga}}\right)$ is

$$
\varepsilon(0 /-)=E_{\mathrm{f}}\left[\mathrm{Mg}_{\mathrm{Ga}}^{-}, \Delta \varepsilon_{\mathrm{F}}=0\right]-E_{\mathrm{f}}\left[\mathrm{Mg}_{\mathrm{Ga}}^{0}\right] \text {. }
$$

The stability of STHs was evaluated using the self-trapping energy $E_{\mathrm{ST}}$, corresponding to the position of $\varepsilon(+/ 0)$ associated with each STH. For a self-trapped, localized hole $\eta^{+}$, the reference neutral state is merely the perfect crystal whose (defect) formation energy is defined to be zero by Eq. (1). Then $E_{\mathrm{ST}}$ or $\varepsilon(+/ 0)$ is given as

$$
E_{\mathrm{ST}}=\varepsilon(+/ 0)=-E_{\mathrm{f}}\left[\eta^{+}, \Delta \varepsilon_{\mathrm{F}}=0\right] .
$$

There is no addition or removal of atoms for $\eta^{+}$and, therefore, the fourth term on the right side of Eq. (1) is absent. Using Eq. (1), Eq. (4) can be deformed as

$$
\varepsilon(+/ 0)=-\left(E\left[\eta^{+}\right]+E_{\text {corr }}\left[\eta^{+}\right]-E_{\mathrm{p}}+\varepsilon_{\mathrm{VBM}}\right),
$$

where $E\left[\eta^{+}\right]$is the total energy of the supercell containing a STH.

\section{B. Generalized Koopmans' theorem}

On the basis of the gKT, the following condition should be satisfied when an electron is removed from the $i$ th occupied single-particle energy level in an $N$-electron system $\varepsilon_{i}(N)$ [52],

$$
E(N)-E(N-1)=\varepsilon_{i}(N),
$$

where $E(N)$ denotes the total energy of the $N$-electron system. When an electron is added to the $i$ th unoccupied energy level in an $(N-1)$-electron system, the $\mathrm{gKT}$ requires

$$
E(N)-E(N-1)=\varepsilon_{i}(N-1) .
$$

From the above, we obtain

$$
E(N)-E(N-1)=\varepsilon_{i}(N)=\varepsilon_{i}(N-1) .
$$

If $\varepsilon_{i}(N-1)$ shifts upward (downward) by electron addition, i.e., $\varepsilon_{i}(N-1)<\varepsilon_{i}(N)\left(\varepsilon_{i}(N-1)>\varepsilon_{i}(N)\right)$, the functional has convex (concave) nature, deviating from the gKT condition.

In this paper, these gKT-relevant quantities were investigated for STHs and substitutional $\mathrm{Mg}$ and $\mathrm{N}$ impurities using a supercell approach, including electrostatic corrections for both total and single-particle energies associated with charged species.

\section{Computational details}

The first-principles calculations were performed using the projector augmented-wave (PAW) method [70] with the HSE range-separated hybrid functional, as implemented in the 
VASP code [56,71,72]. We used PAW data sets with radial cutoffs of $1.40,0.82,1.52$, and $0.74 \AA$ for $\mathrm{Ga}, \mathrm{O}, \mathrm{Mg}$, and $\mathrm{N}$, respectively. Ga $4 s$ and $4 p, \mathrm{O} 2 s$ and $2 p, \mathrm{Mg} 3 s$, and $\mathrm{N}$ $2 s$ and $2 p$ were described as valence electrons. The $\mathrm{Ga} 3 d$ electrons were treated as core electrons; a test STH calculation for $\beta-\mathrm{Ga}_{2} \mathrm{O}_{3}$ showed that the self-trapping energy changed by only $0.01 \mathrm{eV}$ when the $\mathrm{Ga} 3 d$ electrons were included in the valence states. The Fock-exchange mixing parameter, $\alpha$, was set to 0.35 and a screening parameter of $\mu=0.21 \AA^{-1}$ was used in the $\operatorname{HSE}(\alpha, \mu)$ functional, as this set of parameter values has been reported to reproduce an experimental band gap of $\beta-\mathrm{Ga}_{2} \mathrm{O}_{3}$ (4.9 eV [2]) [12,13]; the dependence of the gKT-relevant quantities on $\alpha$ and $\mu$ was also investigated. The lattice vectors and internal atomic coordinates of the unit cells for the four $\mathrm{Ga}_{2} \mathrm{O}_{3}$ polymorphs were relaxed until the residual atomic forces and stresses converged to less than $0.01 \mathrm{eV} / \AA$ and $0.03 \mathrm{GPa}$, respectively. $\Gamma$-centered $k$-point meshes of $6 \times$ $6 \times 6$ for the $\alpha$ phase, $8 \times 8 \times 4$ for $\beta, 4 \times 4 \times 4$ for $\delta$, and $4 \times 2 \times 2$ for $\varepsilon$ were used with a plane-wave cutoff energy of $520 \mathrm{eV}$ in the geometry optimization. The band structure and density of states (DOS) were then evaluated using a plane-wave cutoff energy of $400 \mathrm{eV}$. For DOS calculations, we used $k$-point meshes with double the density.

STHs and acceptor impurities were modeled with 120atom supercells for the $\alpha$ and $\beta$ phases, and 80-atom supercells for $\delta$ and $\varepsilon$. In the case of STHs, an electron was removed from a supercell, and random small atomic displacements were applied around an oxygen site. Internal atomic coordinates were relaxed under the fixed lattice vectors until the atomic forces became smaller than $0.05 \mathrm{eV} / \AA$. We used a $2 \times 2 \times 2 k$-point mesh and a plane-wave cutoff energy of $400 \mathrm{eV}$, and considered spin polarization in these supercell calculations.

The cell-size correction term for electrostatic interactions $E_{\text {corr }}\left[D^{q}\right]$ in Eqs. (1) and (5) was evaluated using the extended Freysoldt-Neugebauer-Van de Walle (extended-FNV) scheme $[68,69]$, where anisotropic screening effects were considered using dielectric tensors [69]. Such spurious electrostatic interactions have also been reported to affect single-particle levels induced by charged defects [73-76]. Therefore, corrections were applied to the relevant single-particle levels using a scheme reported by Chen and Pasquarello [76], where a correction term for $D^{q}$ is given using the total energy correction:

$$
\varepsilon_{\text {corr }}\left[D^{q}\right]=-\frac{2}{q} E_{\text {corr }}\left[D^{q}\right] .
$$

We used the extended-FNV correction energy for $E_{\text {corr }}\left[D^{q}\right]$ as in the case of the total energy correction. The long-range electrostatic screening effects in $E_{\text {corr }}\left[D^{q}\right]$ were described using dielectric tensors involving both ionic and electronic contributions for charged STHs and impurities with relaxed geometries [69]. Only electronic contributions were used to evaluate gKT-relevant quantities at fixed geometries, for which potential reference states were constructed by adding an electron to a STH or taken to be a neutral acceptor impurity. The ionic contributions of the dielectric tensors were calculated using the Perdew-Burke-Ernzerhof (PBE) GGA functional [77] based on density functional perturbation theory $[78,79]$, while their electronic contributions were obtained using $\operatorname{HSE}(0.35,0.21)$ with a finite-electric-field approach
[80]. The effects of the cell-size corrections are discussed in the Appendix.

\section{RESULTS AND DISCUSSION}

\section{A. Bulk electronic structure}

The electronic structure of $\beta-\mathrm{Ga}_{2} \mathrm{O}_{3}$ has been widely investigated using a variety of approximations to the exchangecorrelation [81-83]; however, there are few or no reports on the other polymorphs at the hybrid functional level or based on many-body perturbation theory. Furthmüller and Bechstedt recently reported the quasiparticle band structures and DOS for $\alpha$ - and $\beta-\mathrm{Ga}_{2} \mathrm{O}_{3}$ [83]. They also calculated these properties for $\delta$ - and $\varepsilon-\mathrm{Ga}_{2} \mathrm{O}_{3}$ but at the GGA level. We use the aforementioned $\operatorname{HSE}(0.35,0.21)$ in this section to discuss the bulk electronic properties of the four $\mathrm{Ga}_{2} \mathrm{O}_{3}$ polymorphs.

The crystal structures and coordination environments of the four polymorphs are summarized in Fig. 1 and Table I. $\alpha-\mathrm{Ga}_{2} \mathrm{O}_{3}$ has a corundum structure [Fig. 1(a)] with space group $R \overline{3} c$ and only one nonequivalent site for each of $\mathrm{Ga}$ and $\mathrm{O}$ : the sixfold-coordinated $\mathrm{Ga}$ site and the fourfoldcoordinated $\mathrm{O}$ site. The monoclinic $\beta-\mathrm{Ga}_{2} \mathrm{O}_{3}$ [Fig. 1(b)] with space group $C 2 / m$ has two nonequivalent $\mathrm{Ga}$ sites: $\mathrm{Ga} 1$ is the sixfold-coordinated site, while $\mathrm{Ga} 2$ is the fourfold. In addition, there are three nonequivalent $\mathrm{O}$ sites, which are threefold-coordinated $\mathrm{O} 1$ and $\mathrm{O} 3$ sites, while $\mathrm{O} 2$ is the fourfold. $\delta-\mathrm{Ga}_{2} \mathrm{O}_{3}$ crystallizes in a bixbyite structure [Fig. 1(c)] with space group $I a \overline{3}$. It has two nonequivalent $\mathrm{Ga}$ sites, where both $\mathrm{Ga} 1$ and $\mathrm{Ga} 2$ are sixfold-coordinated sites, and only one nonequivalent fourfold-coordinated $\mathrm{O}$ site. The orthorhombic phase with space group $P n a 2_{1}$, called $\varepsilon-\mathrm{Ga}_{2} \mathrm{O}_{3}$ [Fig. 1(d)], has four nonequivalent $\mathrm{Ga}$ sites: $\mathrm{Ga} 1$ and $\mathrm{Ga} 2$ are fourfold- and sixfold-coordinated sites, respectively, while both $\mathrm{Ga} 3$ and (a)

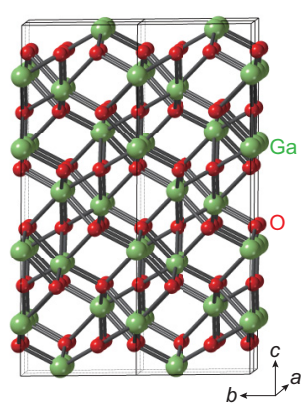

(c)

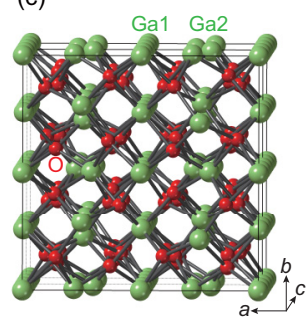

(b)

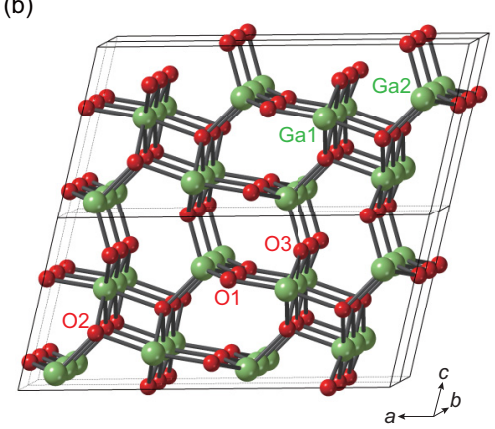

(d)

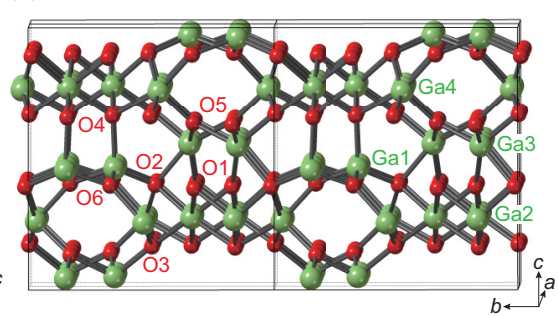

FIG. 1. Crystal structures for (a) $\alpha-\mathrm{Ga}_{2} \mathrm{O}_{3}$, (b) $\beta-\mathrm{Ga}_{2} \mathrm{O}_{3}$, (c) $\delta-\mathrm{Ga}_{2} \mathrm{O}_{3}$, and (d) $\varepsilon-\mathrm{Ga}_{2} \mathrm{O}_{3}$. Green and red balls indicate $\mathrm{Ga}$ and $\mathrm{O}$ atoms, respectively. Each nonequivalent site is described near the balls (see the text and Table I for details). 
TABLE I. Coordination number of each nonequivalent site in the four $\mathrm{Ga}_{2} \mathrm{O}_{3}$ polymorphs. Breakdown for the coordinating atomic sites is provided in the parentheses.

\begin{tabular}{lcccc}
\hline \hline Phase & Cation site & Coordination number & Anion site & Coordination number \\
\hline$\alpha$ & $\mathrm{Ga}$ & 6 & $\mathrm{O}$ & 4 \\
$\beta$ & $\mathrm{Ga} 1$ & $6(\mathrm{O} 1, \mathrm{O} 2 \times 3, \mathrm{O} 3 \times 2)$ & $\mathrm{O} 1$ & $3(\mathrm{Ga} 1, \mathrm{Ga} 2 \times 2)$ \\
& $\mathrm{Ga} 2$ & $4(\mathrm{O} 1 \times 2, \mathrm{O} 2, \mathrm{O} 3)$ & $\mathrm{O} 2$ & $3(\mathrm{Ga} 1 \times 3, \mathrm{Ga} 2)$ \\
& & & $\mathrm{O} 3$ & $4(\mathrm{Ga} 1 \times 2, \mathrm{Ga} 2)$ \\
$\delta$ & $\mathrm{Ga} 1$ & 6 & $\mathrm{O}$ & $3(\mathrm{Ga} 1, \mathrm{Ga} 2 \times 3)$ \\
& $\mathrm{Ga} 2$ & 6 & $\mathrm{O} 1$ & $\mathrm{O} 2 \times 2, \mathrm{Ga} 3)$ \\
& $\mathrm{Ga} 1$ & $4(\mathrm{O} 2, \mathrm{O} 4, \mathrm{O} 6 \times 2)$ & $\mathrm{O} 2$ & $3(\mathrm{Ga} 1, \mathrm{Ga} 2, \mathrm{Ga} 3, \mathrm{Ga} 4)$ \\
& $\mathrm{Ga} 2$ & $6(\mathrm{O} 1 \times 2, \mathrm{O} 2, \mathrm{O} 3, \mathrm{O} 4 \times 2)$ & $\mathrm{O} 3$ & $4(\mathrm{Ga} 1, \mathrm{Ga} 2 \times 2, \mathrm{Ga} 4)$ \\
& $\mathrm{Ga} 3$ & $5(\mathrm{O} 1, \mathrm{O} 2, \mathrm{O} 3, \mathrm{O} 5 \times 2)$ & $3(\mathrm{Ga} 3 \times 2, \mathrm{Ga} 4)$ \\
& $\mathrm{Ga} 4$ & $5(\mathrm{O} 2, \mathrm{O} 3, \mathrm{O} 4, \mathrm{O} 5, \mathrm{O} 6)$ & $\mathrm{O} 5$ & $3(\mathrm{Ga} 1 \times 2, \mathrm{Ga} 4)$ \\
\hline \hline
\end{tabular}

Ga4 are fivefold-coordinated sites. It also has six nonequivalent $\mathrm{O}$ sites: $\mathrm{O} 1, \mathrm{O} 3, \mathrm{O} 5$, and $\mathrm{O} 6$ are threefold-coordinated sites, while $\mathrm{O} 2$ and $\mathrm{O} 4$ are fourfold.

Figure 2 shows the band structures for the four $\mathrm{Ga}_{2} \mathrm{O}_{3}$ polymorphs, and the direct and indirect band gap values are summarized in Table II. In all polymorphs, the conduction band minima are at the $\Gamma$ points, while the VBMs are at low symmetry points. The band gap of $\beta-\mathrm{Ga}_{2} \mathrm{O}_{3}$ calculated using $\operatorname{HSE}(0.35,0.21)$ well reproduces the experimental value of $4.9 \mathrm{eV}$ [2], as previously reported [12,13]. A wide range of experimental band gap values has been reported for $\alpha-\mathrm{Ga}_{2} \mathrm{O}_{3}(5.0-5.6 \mathrm{eV}$; Refs. [33,35,85]), and our results using $\operatorname{HSE}(0.35,0.21)$ fall within this range. $\alpha$ - and $\delta-\mathrm{Ga}_{2} \mathrm{O}_{3}$ have indirect band gaps that are 0.23 and $0.15 \mathrm{eV}$ smaller than the (a)

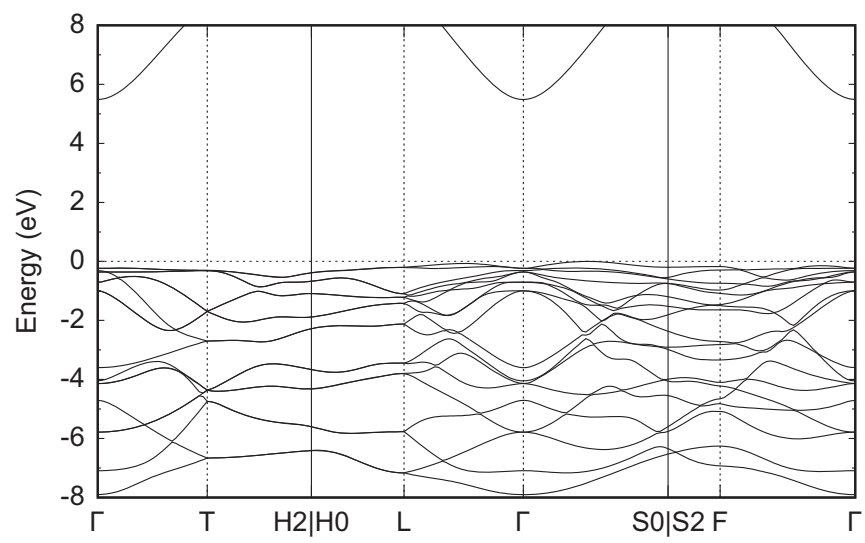

(c)

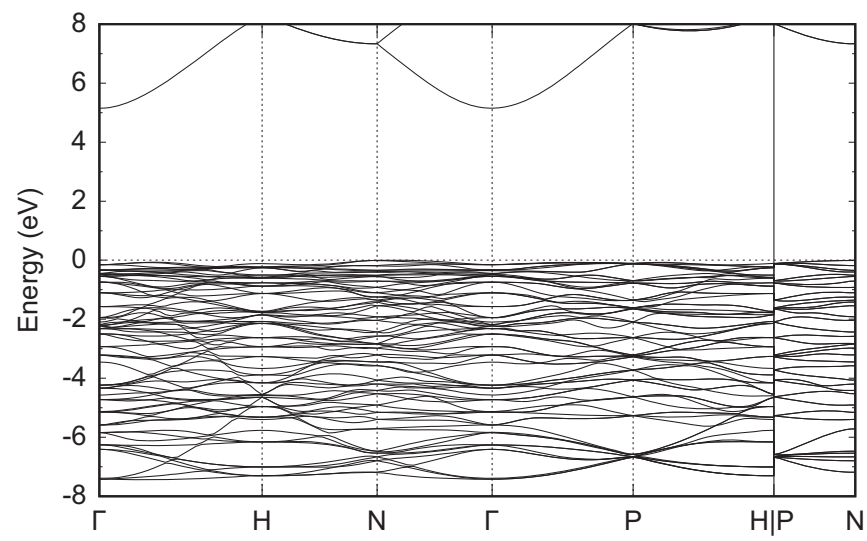

(b)

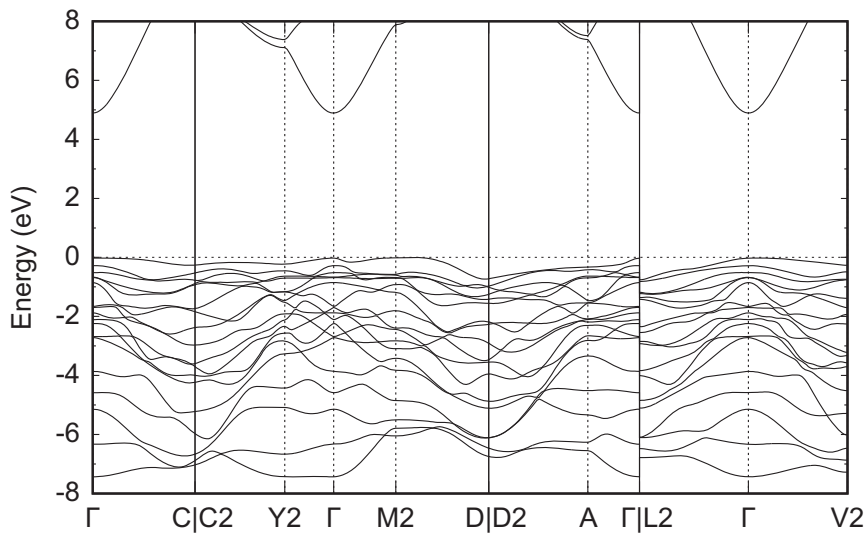

(d)

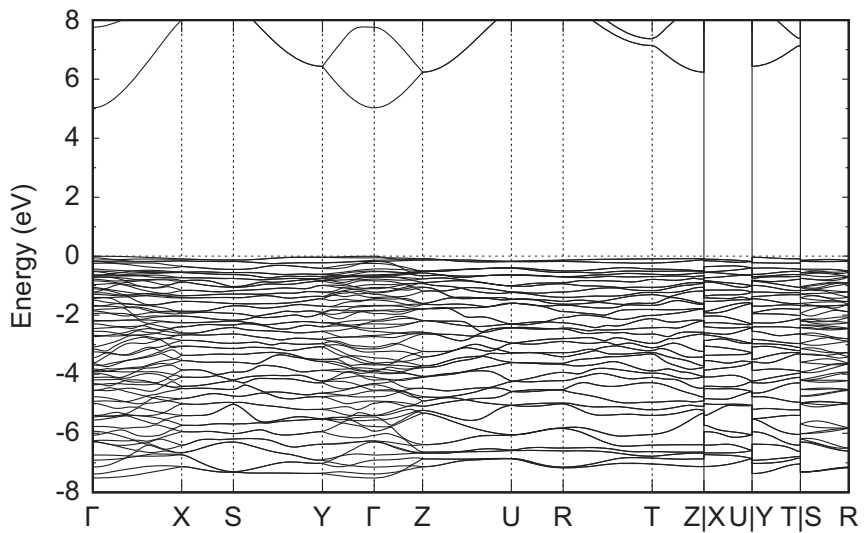

FIG. 2. Band structures for (a) $\alpha-\mathrm{Ga}_{2} \mathrm{O}_{3}$, (b) $\beta-\mathrm{Ga}_{2} \mathrm{O}_{3}$, (c) $\delta-\mathrm{Ga}_{2} \mathrm{O}_{3}$, and (d) $\varepsilon-\mathrm{Ga}_{2} \mathrm{O}_{3}$ obtained using $\mathrm{HSE}(0.35,0.21)$. The valence band maxima are aligned to the zero energies. The band paths conform to Ref. [84]. 
TABLE II. Direct and indirect band gaps and electron effective masses for the four $\mathrm{Ga}_{2} \mathrm{O}_{3}$ polymorphs obtained using $\mathrm{HSE}(0.35$, 0.21$)$. Experimental values are also shown for comparison. The electron effective masses are calculated along the paths from the $\Gamma$ point to relevant high-symmetry points; however, they are almost isotropic with values of $0.26 m_{0}-0.28 m_{0}$, where $m_{0}$ is the electron rest mass, and the lowest values are shown in the table.

\begin{tabular}{|c|c|c|c|c|c|}
\hline \multirow[b]{2}{*}{ Phase } & \multicolumn{3}{|c|}{ Band gap (eV) } & \multicolumn{2}{|c|}{ Electron effective mass $\left(m_{0}\right)$} \\
\hline & Direct & Indirect & Experimental & This work & Experimental \\
\hline$\alpha$ & 5.72 & 5.49 & 4.98 [85], 5.3 [33], $5.61[35]$ & 0.26 & - \\
\hline$\beta$ & 4.92 & 4.89 & $4.9[2]$ & 0.27 & $0.28[86]$ \\
\hline$\delta$ & 5.30 & 5.15 & - & 0.27 & - \\
\hline$\varepsilon$ & 5.03 & 5.03 & - & 0.26 & - \\
\hline
\end{tabular}

direct gaps at the $\Gamma$ point, respectively [Figs. 2(a) and 2(c), and Table II]. $\beta$ - and $\varepsilon-\mathrm{Ga}_{2} \mathrm{O}_{3}$ also have indirect-type band structures, but the energy differences between their indirect and direct band gaps are only 30 and $1.1 \mathrm{meV}$, respectively [Figs. 2(b) and 2(d), and Table II].

According to Ref. [83], $\alpha-\mathrm{Ga}_{2} \mathrm{O}_{3}$ has an indirect gap of $5.39 \mathrm{eV}$ and the energy difference from the direct gap is $-0.24 \mathrm{eV}$ at the $G_{0} W_{0} @ \mathrm{HSE03}$ level. This is close to our $\operatorname{HSE}(0.35,0.21)$ results $(5.49 \mathrm{eV}$ and $-0.23 \mathrm{eV}$, respectively). $\beta-\mathrm{Ga}_{2} \mathrm{O}_{3}$ has a direct gap of $5.04 \mathrm{eV}$ with $G_{0} W_{0} @$ HSE03, where the energy difference from the indirect gap is $-8.0 \mathrm{meV}$, while our results show an indirect gap of $4.89 \mathrm{eV}$ and a $30 \mathrm{meV}$ larger direct gap. We expect this small discrepancy between $G_{0} W_{0} @ \operatorname{HSE} 03$ and $\operatorname{HSE}(0.35,0.21)$ has an insignificant effect on our discussion.

In all polymorphs, the valence bands are mainly derived from $\mathrm{O} 2 p$ orbitals and show small energy dispersions, and the conduction bands are predominantly made up of Ga $4 s$ orbitals with free-electron-like dispersions. We calculated the effective masses of electrons using parabolic fitting of conduction bands along the $\Gamma$ point to relevant high-symmetry points. In all directions and in all polymorphs, almost equal values between $0.26 m_{0}$ and $0.28 m_{0}$ were obtained as summarized in Table II, where $m_{0}$ is the electron rest mass. The values for $\beta-\mathrm{Ga}_{2} \mathrm{O}_{3}$ are in good agreement with previous experimental [86] and theoretical $[82,83]$ reports. An analysis of atomic-site projected DOS revealed that the upper valence bands are mainly formed by $\mathrm{O} 2 p$ orbitals and the lower valence bands by hybridization of $\mathrm{Ga} 4 s$ with $\mathrm{O} 2 p$ orbitals, while $\mathrm{Ga} 4 p$ orbitals are almost evenly spread in the valence bands in all the polymorphs.

\section{B. Quantities relevant to generalized Koopmans' theorem}

The dependence of the gKT-relevant quantities on the Fock-exchange mixing parameter $\alpha$ and screening parameter $\mu$ in the HSE functional was investigated by taking the $\mathrm{STH}$ on the $\mathrm{O} 3$ site in $\beta-\mathrm{Ga}_{2} \mathrm{O}_{3}$ as an example. The STH geometry and the cell-size correction terms for total energy, $E_{\text {corr }}\left[\eta^{+}\right]$, and single-particle energy, $\varepsilon_{\text {corr }}\left[\eta^{+}\right]$, obtained using $\operatorname{HSE}(0.35,0.21)$, were applied to a range of parameter values; effectiveness of the cell-size corrections is demonstrated in the Appendix. Figure 3(a) shows the dependence of $E(N)-$ $E(N-1)$ for the STH and its single-particle level $\left[\varepsilon_{\mathrm{ST}}(N-1)\right.$ and $\left.\varepsilon_{\mathrm{ST}}(N)\right]$ on $\alpha$, where $\mu$ is fixed at $0.21 \AA^{-1}$. When using $\operatorname{HSE}(0.35,0.21)$, which reproduces the band gap of $\beta-\mathrm{Ga}_{2} \mathrm{O}_{3}$ well as mentioned above, $\varepsilon_{\mathrm{ST}}(N-1)$ slightly shifts upward when the electron is added $\left[\varepsilon_{\mathrm{ST}}(N)\right]$. In other words, the functional shows convex behavior, promoting hole delocalization in small measure. We find that $\varepsilon_{\mathrm{ST}}(N-1)$ and $\varepsilon_{\mathrm{ST}}(N)$ almost linearly increase and decrease with increasing

(a)

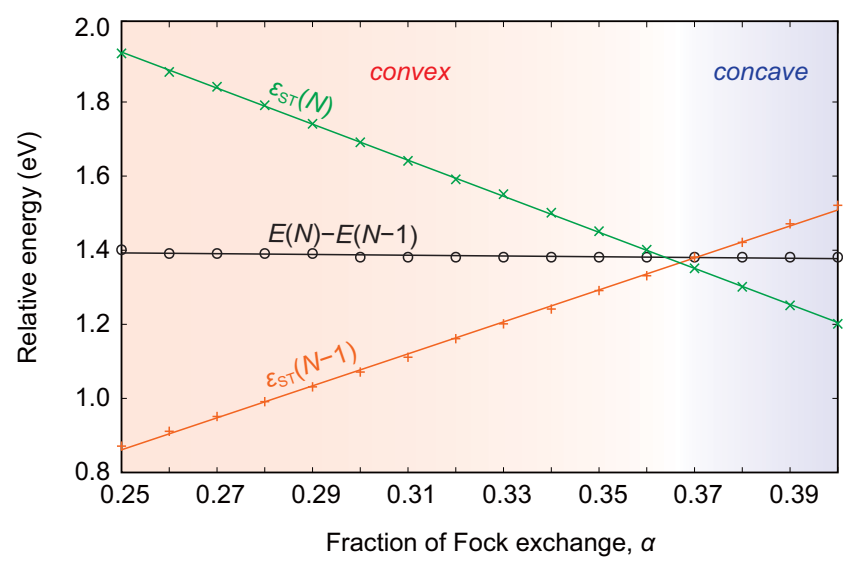

(b)

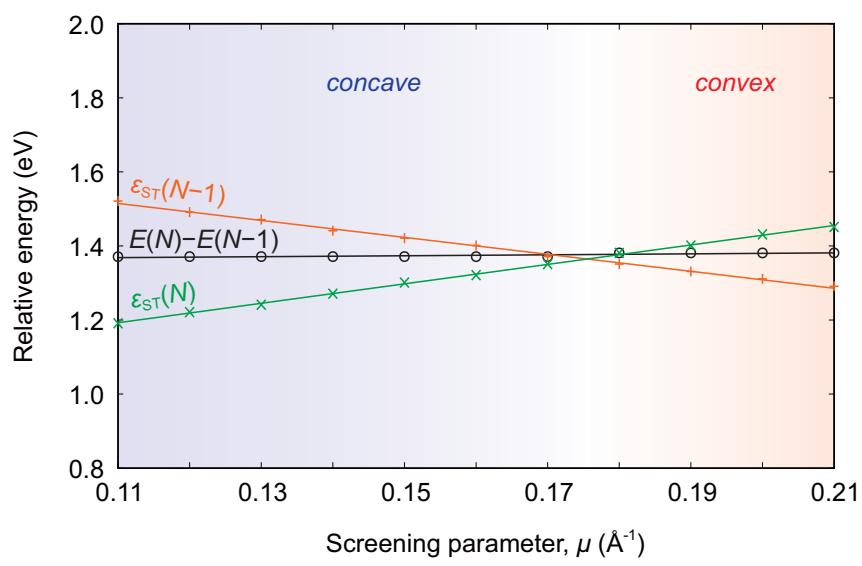

FIG. 3. Total energy difference between the neutral supercell with STH geometry and a positively charged supercell with a STH, $E(N)-E(N-1)$, and STH single-particle levels without and with an electron addition, $\varepsilon_{\mathrm{ST}}(N-1)$ and $\varepsilon_{\mathrm{ST}}(N)$, respectively, for the $\mathrm{STH}$ on the $\mathrm{O} 3$ site in $\beta-\mathrm{Ga}_{2} \mathrm{O}_{3}$ for (a) $\mathrm{HSE}(\alpha, 0.21)$ and (b) $\operatorname{HSE}(0.35, \mu)$. Solid lines show linear fits to the data. STH geometry and corrections for total energy, $E_{\text {corr }}\left[\eta^{+}\right]$, and singleparticle energy, $\varepsilon_{\text {corr }}\left[\eta^{+}\right]$, obtained using $\operatorname{HSE}(0.35,0.21)$ are applied to a range of parameter values. The vertical axes are relative energy to the VBM obtained using $\operatorname{HSE}(0.35,0.21)$. 
TABLE III. Indirect band gaps of $\beta-\mathrm{Ga}_{2} \mathrm{O}_{3}$ and $E(N)-E(N-1), \varepsilon_{\mathrm{ST}}(N), \varepsilon_{\mathrm{ST}}(N-1)$, and $\varepsilon_{\mathrm{ST}}(N)-\varepsilon_{\mathrm{ST}}(N-1)$ of its STH on the O3 site for selected parameter values $(\alpha, \mu)$ in the HSE functional. STH geometry and corrections for total energy, $E_{\text {corr }}\left[\eta^{+}\right]$, and single-particle energy, $\varepsilon_{\text {corr }}\left[\eta^{+}\right]$, obtained using $\operatorname{HSE}(0.35,0.21)$ are applied in all cases. Positive and negative values for $\varepsilon_{\mathrm{ST}}(N)-\varepsilon_{\mathrm{ST}}(N-1)$ mean convex and concave, respectively, for curves of total energy versus fractional electron number $N$. The gKT-relevant quantities are relative values to the VBM obtained using $\operatorname{HSE}(0.35,0.21)$. All values are in $\mathrm{eV}$.

\begin{tabular}{lcccrr}
\hline \hline$(\alpha, \mu)$ & Indirect gap & $E(N)-E(N-1)$ & $\varepsilon_{\mathrm{ST}}(N)$ & $\varepsilon_{\mathrm{ST}}(N-1)$ & $\varepsilon_{\mathrm{ST}}(N)-\varepsilon_{\mathrm{ST}}(N-1)$ \\
\hline$(0.35,0.21)$ & 4.89 & 1.38 & 1.45 & 1.29 & 0.16 \\
$(0.37,0.21)$ & 5.07 & 1.38 & 1.35 & 1.38 & -0.03 \\
$(0.35,0.17)$ & 5.06 & 1.37 & 1.35 & 1.37 & -0.02 \\
$(0.26,0.00)$ & 4.83 & 1.38 & 1.46 & 1.30 & 0.16 \\
\hline \hline
\end{tabular}

$\alpha$, respectively, while $E(N)-E(N-1)$ is nearly independent of $\alpha$. The linear fits of the three quantities approximately intersect at $\alpha=0.37$. We also find that $\varepsilon_{\mathrm{ST}}(N-1)$ and $\varepsilon_{\mathrm{ST}}(N)$ have nearly linear dependencies on the screening parameter $\mu$ when $\alpha=0.35$, while $E(N)-E(N-1)$ is almost independent of $\mu$, and these three approximately intersect at $\mu=0.17 \AA^{-1}$ [Fig. 3(b)]. Consequently, two hybrid functionals, $\operatorname{HSE}(0.37,0.21)$ and $\operatorname{HSE}(0.35,0.17)$, satisfy the gKT for the STH level when each of $\alpha$ and $\mu$ is changed from that of $\operatorname{HSE}(0.35,0.21)$. Using these parameter values, we optimized lattice vectors and internal atomic coordinates, and calculated the band structure for the $\beta-\mathrm{Ga}_{2} \mathrm{O}_{3}$ primitive cell. $\operatorname{HSE}(0.37,0.21)$ and $\operatorname{HSE}(0.35,0.17)$ are found to yield band gaps of 5.07 and $5.06 \mathrm{eV}$, respectively, while the experimental value is $4.9 \mathrm{eV}$ [2]. These parameter values thus satisfy the gKT for the STH level but overestimate the band gap of $\beta-\mathrm{Ga}_{2} \mathrm{O}_{3}$ by $\sim 0.2 \mathrm{eV}$.

Table III shows the indirect band gap, $E(N)-E(N-1)$, $\varepsilon_{\mathrm{ST}}(N), \quad \varepsilon_{\mathrm{ST}}(N-1)$, and $\varepsilon_{\mathrm{ST}}(N)-\varepsilon_{\mathrm{ST}}(N-1)$ for selected parameter values. Deák et al. [18] reported that $\operatorname{HSE}(0.26,0.00)$ reproduces the experimental band gap and satisfies the $\mathrm{gKT}$ for $\mathrm{Ga}$ and $\mathrm{O}$ vacancies in $\beta-\mathrm{Ga}_{2} \mathrm{O}_{3}$. The resultant functional is close to $\mathrm{PBE} 0$, which corresponds to $\operatorname{HSE}(0.25,0.00)$ [87]. Furthermore, they found that the theoretical thermodynamic transition levels for these vacancies and STHs quantitatively agree with experiment, although their study did not check for gKT conditions for the STHs. It has been reported that satisfying the gKT depends on defect species, particularly the spatial extent of the defect states $[65,67]$. In our STH calculation, $\operatorname{HSE}(0.26,0.00)$ shows almost the same convex behavior and band gap value as those for $\operatorname{HSE}(0.35,0.21)$.

Moving back to Fig. 3, the energy difference between the neutral supercell with a STH geometry and the supercell containing the STH, $E(N)-E(N-1)$, is almost independent of $\alpha$ and $\mu$. Actually, $E(N)-E(N-1)$ corresponds to the absolute position of a thermodynamic transition level excluding the atomic relaxation contribution. This shows that the calculated STH level or self-trapping energy is robust for a wide range of $\alpha$ and $\mu$ values, as long as an appropriate STH geometry and electronic structure are obtained and the absolute VBM position is well reproduced.

The ionization potential (IP), which is the VBM with respect to the vacuum level, is typically used to discuss the absolute VBM position, although the IP depends on the surface orientation and structure because of the surface dipole contribution [88-92]. To our knowledge, the IPs of $\mathrm{Ga}_{2} \mathrm{O}_{3}$ surfaces have not been well established experimentally, although the electron affinity has been estimated to be $4.00 \pm$ $0.05 \mathrm{eV}$ at an $\mathrm{Au} / \beta-\mathrm{Ga}_{2} \mathrm{O}_{3}(100)$ Schottky junction [93]. Given that the band gap of $\beta-\mathrm{Ga}_{2} \mathrm{O}_{3}$ is $4.9 \mathrm{eV}$ [2], the IP is estimated at $8.9 \mathrm{eV}$. Previous theoretical studies on other oxides such as $\mathrm{ZnO}$ and $\mathrm{MgO}$ have shown that tuning $\alpha$ and/or $\mu$ in the HSE functional to reproduce band gaps also improves IPs $[94,95]$. We theoretically determine the IP of $\beta-\mathrm{Ga}_{2} \mathrm{O}_{3}$ to check whether it is improved by band-gap tuning in this case. We use a combination of bulk and slab-vacuum models as typically done in the IP evaluation [25], the latter of which is for a $\beta-\mathrm{Ga}_{2} \mathrm{O}_{3}(100)$ surface with 40 atoms and the slab and vacuum thickness over $20 \AA$ and constructed on the basis of the procedure described in Ref. [96]. Since surface geometry optimization using the hybrid functional is computationally demanding, bulk and surface geometries are optimized using the PBEsol functional [97], which improves the lattice constants of $\beta-\mathrm{Ga}_{2} \mathrm{O}_{3}$ over the standard PBE functional [98] and yields values closer to those of $\operatorname{HSE}(0.35,0.21)$. Then, the lattice constants of the bulk and slab-vacuum models are scaled using those of $\operatorname{HSE}(0.35,0.21)$ with the internal coordinates kept. Together with the bulk $\operatorname{HSE}(0.35,0.21)$ result, we obtain an IP value of $8.7 \mathrm{eV}$ in good agreement with the experimental value of $8.9 \mathrm{eV}$ estimated above. This verifies that $\operatorname{HSE}(0.35,0.21)$ well reproduces the IP in addition to the band gap. We deem the STH geometry and electronic structures are close between $\operatorname{HSE}(0.37,0.21)$ and $\operatorname{HSE}(0.35,0.21)$, which are rationalized in terms of the gKT condition and the band structure, respectively, and investigate STHs and substitutional $\mathrm{Mg}$ and $\mathrm{N}$ impurities in the four $\mathrm{Ga}_{2} \mathrm{O}_{3}$ polymorphs using $\mathrm{HSE}(0.35,0.21)$.

\section{Self-trapped holes and acceptor impurities}

Figure 4 shows the relaxed atomic configurations and isosurfaces of the squared wave functions for the STHs on selected nonequivalent $\mathrm{O}$ sites in the four $\mathrm{Ga}_{2} \mathrm{O}_{3}$ polymorphs obtained using $\operatorname{HSE}(0.35,0.21)$. The STH is localized on an $\mathrm{O}$ site with an $\mathrm{O} 2 p$-like state in $\alpha-\mathrm{Ga}_{2} \mathrm{O}_{3}$ [Fig. 4(a)] with $E_{\mathrm{ST}}$ of $0.54 \mathrm{eV}$. The four Ga neighbors around the STH site have averagely relaxed outward by $0.13 \AA$, which can be attributed to the repulsive interaction between the positively charged hole and $\mathrm{Ga}$ ions. The $\mathrm{STH}$ in $\beta-\mathrm{Ga}_{2} \mathrm{O}_{3}$ shows particular behavior on each site. A hole is trapped by an $\mathrm{O}$ atom on the $\mathrm{O} 3$ site [Fig. 4(c)] with average outward relaxation of the Ga neighbors by $0.16 \AA$. At the $\mathrm{O} 1$ site, a hole is distributed among two $\mathrm{O}$ sites [Fig. 4(b)], which have relaxed 

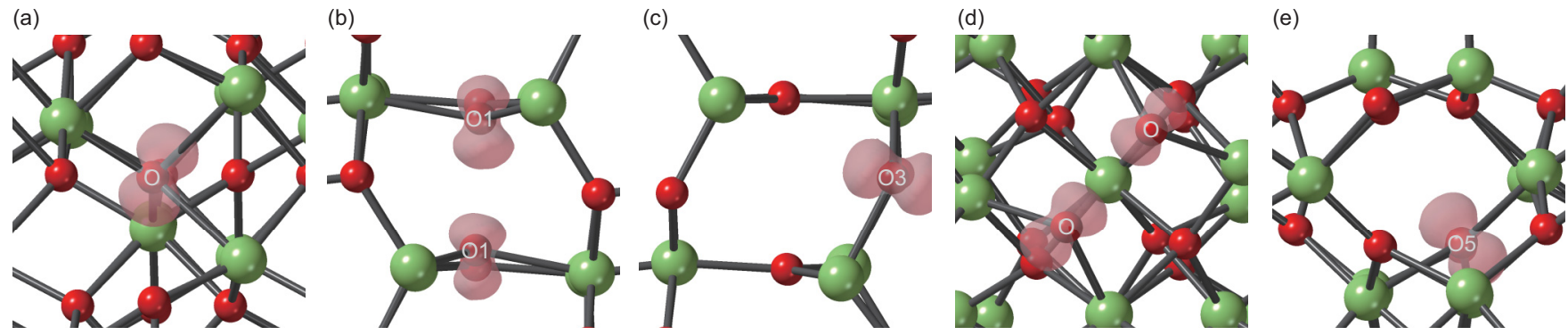

FIG. 4. Relaxed atomic configurations and isosurfaces of the squared wave functions for the $\mathrm{STHs}$ on (a) the $\mathrm{O}$ site in $\alpha$-Ga $\mathrm{O}_{3}$, (b) the $\mathrm{O} 1$ site and (c) the $\mathrm{O} 3$ site in $\beta-\mathrm{Ga}_{2} \mathrm{O}_{3}$, (d) the $\mathrm{O}$ site in $\delta-\mathrm{Ga}_{2} \mathrm{O}_{3}$, and (e) the $\mathrm{O} 5$ site in $\varepsilon-\mathrm{Ga}_{2} \mathrm{O}_{3}$. Green and red balls indicate $\mathrm{Ga}$ and $\mathrm{O}$ atoms, respectively. The isosurfaces correspond to $10 \%$ of the maximum value.

by 0.25 and $0.27 \AA$, respectively, to approach each other. Both STHs show nearly the same $E_{\mathrm{ST}}$ values of $0.58 \mathrm{eV}$. To our knowledge, the STH on the $\mathrm{O} 2$ site in $\beta-\mathrm{Ga}_{2} \mathrm{O}_{3}$ has not been reported previously, and we could not stabilize it. In $\delta-\mathrm{Ga}_{2} \mathrm{O}_{3}$, a split STH forms in the same manner as the O1-site STH in $\beta-\mathrm{Ga}_{2} \mathrm{O}_{3}$, and $E_{\mathrm{ST}}$ is estimated to be $0.65 \mathrm{eV}$ [Fig. 4(d)]. Similarly, two $\mathrm{O}$ atoms have approached each other with relaxation by 0.20 and $0.27 \AA$. $\varepsilon-\mathrm{Ga}_{2} \mathrm{O}_{3}$ exhibits a large site dependence of $E_{\mathrm{ST}}$. Although a hole is trapped by a single $\mathrm{O}$ atom in each case [Fig. 4(e) for the $\mathrm{O} 5$ site], $E_{\mathrm{ST}}$ varies widely from 0.46 to $1.08 \mathrm{eV}$ (O1 to O5). The average outward relaxations of the neighboring $\mathrm{Ga}$ atoms for the O1-, O3-, and O5-site STHs are 0.09, 0.15, and $0.16 \AA$, respectively. When there are both threefold- and fourfold-coordinated $\mathrm{O}$ sites as in $\beta$ - and $\varepsilon-\mathrm{Ga}_{2} \mathrm{O}_{3}$, the STHs prefer to be localized on the threefold-coordinated sites with higher $E_{\mathrm{ST}}$. For $\alpha$ - and $\delta$ - $\mathrm{Ga}_{2} \mathrm{O}_{3}$, which have only fourfold-coordinated $\mathrm{O}$ sites, the STHs are stabilized with $E_{\mathrm{ST}}$ comparable to or even greater than on the threefold-coordinated sites in $\beta-\mathrm{Ga}_{2} \mathrm{O}_{3}$. In any case, STHs are stable with high $E_{\mathrm{ST}}$ and, therefore, high hole-mobility would be extremely difficult to obtain for all four $\mathrm{Ga}_{2} \mathrm{O}_{3}$ polymorphs.

In addition to STHs, we investigate the acceptor levels of representative substitutional impurities to obtain further detail of the difficulty of $p$-type doping of $\mathrm{Ga}_{2} \mathrm{O}_{3}$ polymorphs. We focus on $\mathrm{Mg}_{\mathrm{Ga}}$ because it is the most stable acceptor and has the lowest ionization energy (the lowest $0 /-$ thermodynamic transition level) among the group II impurities in $\beta-\mathrm{Ga}_{2} \mathrm{O}_{3}$, according to Ref. [16]. For an anion-site acceptor, we consider $\mathrm{N}$ on $\mathrm{O}\left(\mathrm{N}_{\mathrm{O}}\right)$.

Figure 5 shows acceptor levels for $\mathrm{Mg}_{\mathrm{Ga}}$ and $\mathrm{N}_{\mathrm{O}}$ on nonequivalent sites in the four $\mathrm{Ga}_{2} \mathrm{O}_{3}$ polymorphs relative to respective VBMs. The $+/ 0$ thermodynamic transition levels for the STHs, corresponding to $E_{\mathrm{ST}}$, are also shown for comparison. Our calculated acceptor levels for $\mathrm{Mg}_{\mathrm{Ga}}$ and $\mathrm{N}_{\mathrm{O}}$ in $\beta-\mathrm{Ga}_{2} \mathrm{O}_{3}$ agree well with those reported in Ref. [16]. In Fig. 5, the acceptor levels are very far from the VBM for both $\mathrm{Mg}_{\mathrm{Ga}}$ and $\mathrm{N}_{\mathrm{O}}$ in all polymorphs. This means that $\mathrm{Mg}_{\mathrm{Ga}}$ and $\mathrm{N}_{\mathrm{O}}$ impurities act as deep acceptors and do not essentially contribute to carrier generation in any of the $\mathrm{Ga}_{2} \mathrm{O}_{3}$ polymorphs. The acceptor levels of $\mathrm{Mg}_{\mathrm{Ga}}$ are generally lower than those of $\mathrm{N}_{\mathrm{O}}$, but even the lowest one for $\alpha-\mathrm{Ga}_{2} \mathrm{O}_{3}$ lies $1.07 \mathrm{eV}$ above the VBM. Figure 6 shows the relaxed atomic configurations and isosurfaces of the squared wave functions of selected acceptor states. As reported in Ref. [16], $\mathrm{Mg}_{\mathrm{Ga}}^{0}$ in $\beta-\mathrm{Ga}_{2} \mathrm{O}_{3}$ causes hole trapping by one of the neighboring $\mathrm{O}$ atoms. For such a deep, polaronic acceptor, a released hole would tend to be trapped on an $\mathrm{O}$ site (or two $\mathrm{O}$ sites) with the highest $E_{\mathrm{ST}}$ among the neighboring nonequivalent $\mathrm{O}$ sites; such a neighboring configuration should be energetically favorable in view of electrostatic attractive interaction between a negatively charged acceptor impurity and a positively charged (a)

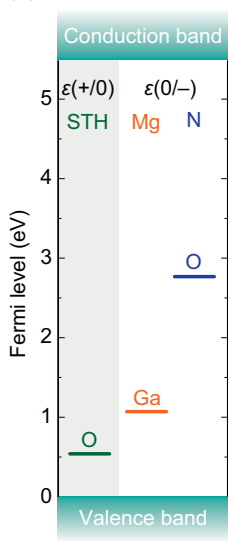

(b)

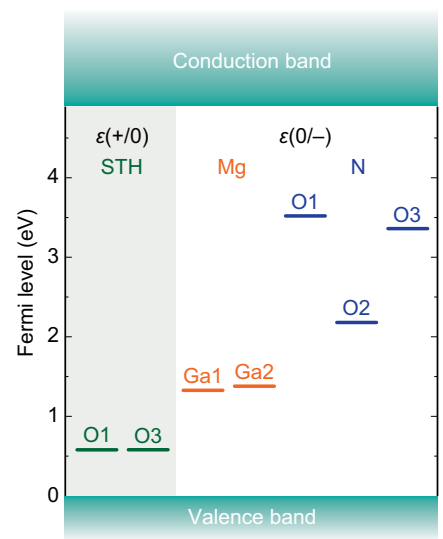

(c)

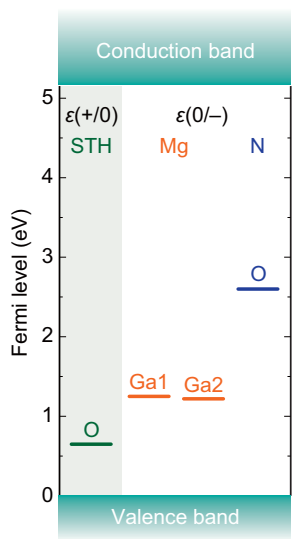

(d)

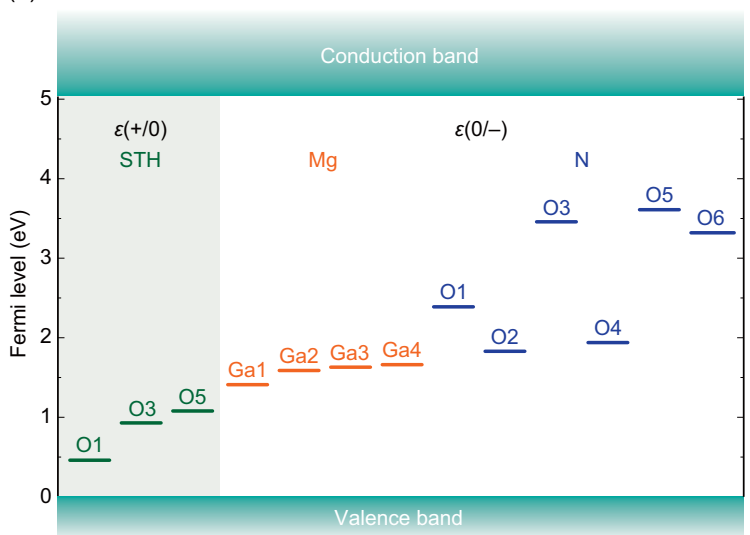

FIG. 5. Thermodynamic transition levels for STHs, $\mathrm{Mg}_{\mathrm{Ga}}$, and $\mathrm{N}_{\mathrm{O}}$ on nonequivalent sites in the four $\mathrm{Ga}_{2} \mathrm{O}_{3}$ polymorphs obtained using $\operatorname{HSE}(0.35,0.21)$ : (a) $\alpha-\mathrm{Ga}_{2} \mathrm{O}_{3}$, (b) $\beta-\mathrm{Ga}_{2} \mathrm{O}_{3}$, (c) $\delta-\mathrm{Ga}_{2} \mathrm{O}_{3}$, and (d) $\varepsilon-\mathrm{Ga}_{2} \mathrm{O}_{3}$. The sites of the STHs and substitutional impurities are described above the levels. Note that the STHs are not stabilized on the $\mathrm{O} 2$ site in $\beta-\mathrm{Ga}_{2} \mathrm{O}_{3}$ or the $\mathrm{O} 2$, $\mathrm{O} 4$, and $\mathrm{O} 6$ sites in $\varepsilon$ - $\mathrm{Ga}_{2} \mathrm{O}_{3}$. 

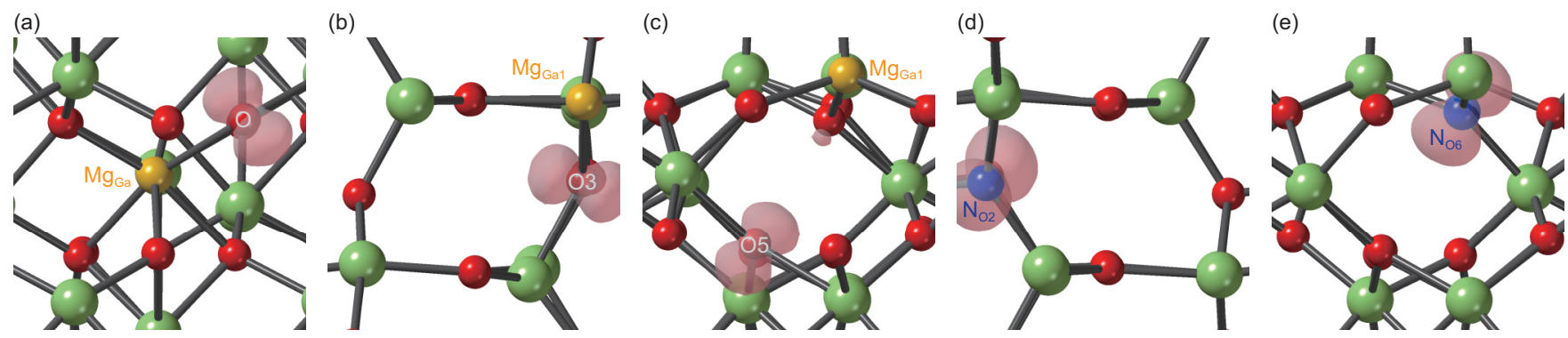

FIG. 6. Relaxed atomic configurations and isosurfaces of the squared wave functions of acceptor states for (a) $\mathrm{Mg}_{\mathrm{Ga}}^{0}$ in $\alpha$ - $\mathrm{Ga}_{2} \mathrm{O}_{3}$, (b) $\mathrm{Mg}_{\mathrm{Gal}}^{0}$ in $\beta-\mathrm{Ga}_{2} \mathrm{O}_{3}$, (c) $\mathrm{Mg}_{\mathrm{Ga} 1}^{0}$ in $\varepsilon-\mathrm{Ga}_{2} \mathrm{O}_{3}$, (d) $\mathrm{N}_{\mathrm{O} 2}^{0}$ in $\beta-\mathrm{Ga}_{2} \mathrm{O}_{3}$, and (e) $\mathrm{N}_{\mathrm{O} 6}^{0}$ in $\varepsilon-\mathrm{Ga}_{2} \mathrm{O}_{3}$. Green, red, orange, and blue balls indicate $\mathrm{Ga}$, $\mathrm{O}, \mathrm{Mg}$, and $\mathrm{N}$ atoms, respectively. The isosurfaces correspond to $10 \%$ of the maximum value.

hole. This tendency holds for $\mathrm{Mg}_{\mathrm{Ga}}$ in the $\mathrm{Ga}_{2} \mathrm{O}_{3}$ polymorphs with only one exception: $\mathrm{Mg}_{\mathrm{Ga}}$ in $\varepsilon-\mathrm{Ga}_{2} \mathrm{O}_{3}$. Although the Ga1 site in $\varepsilon-\mathrm{Ga}_{2} \mathrm{O}_{3}$ is surrounded by the $\mathrm{O} 2, \mathrm{O} 4$, and two $\mathrm{O} 6$ sites, a hole is mainly localized on a non-neighboring $\mathrm{O} 5$ site [Fig. 6(c)]. As discussed above, the STH cannot be stabilized on the $\mathrm{O} 2, \mathrm{O} 4$, and $\mathrm{O} 6$ sites and localizes on the $\mathrm{O} 5$ site with the highest $E_{\mathrm{ST}}$. The stability of a released hole on the $\mathrm{O} 5$ site appears to overcome the electrostatic attractive interaction in the acceptor-hole neighboring configuration.

To obtain further insight into the electrostatic effect, we calculated the acceptor state for $\mathrm{Na}_{\mathrm{Ga}}^{-1}$ in $\varepsilon-\mathrm{Ga}_{2} \mathrm{O}_{3}$ that caputures a single hole polaron as in the case of $\mathrm{Mg}_{\mathrm{Ga}}^{0}$, where $\mathrm{Na} 3 s$ was treated as a valence electron with a PAW radial cutoff of $1.76 \AA$. As a result, a released hole was trapped on the neighboring but STH nonpreferable site (O6), instead of the $\mathrm{O} 5$ site with the highest $E_{\mathrm{ST}}$. This is most likely due to a larger electrostatic gain than the case of $\mathrm{Mg}_{\mathrm{Ga}}^{0}$. Thus, the acceptor-hole configuration would be determined by the balance between the trapping and electrostatic energies.

The acceptor levels for $\mathrm{Mg}_{\mathrm{Ga}}$ are higher than the STH levels, as shown in Fig. 5. This behavior can also be understood from the electrostatic attractive interaction that stabilizes the released hole. For $\mathrm{Mg}_{\mathrm{Ga}}^{0}$ in $\varepsilon-\mathrm{Ga}_{2} \mathrm{O}_{3}$, the distance between $\mathrm{Mg}_{\mathrm{Ga}}^{-}$and the released hole is longer than those for $\mathrm{Mg}_{\mathrm{Ga}}$ at the other sites, which means the electrostatic attraction becomes weaker. The acceptor level for $\mathrm{Mg}_{\mathrm{Ga} 1}$ is somewhat close to the $+/ 0$ thermodynamic transition level for the STH on the $\mathrm{O} 5$ site, with a $0.33 \mathrm{eV}$ difference; the positions of the thermodynamic transition levels of $\mathrm{Mg}_{\mathrm{Ga}}$ and the STH differ by more than $0.53 \mathrm{eV}$ on the other sites in all $\mathrm{Ga}_{2} \mathrm{O}_{3}$ polymorphs when the acceptor-induced and self-trapped holes are on the same site.

Figures 6(d) and 6(e) show the squared wave functions of the acceptor states for $\mathrm{N}_{\mathrm{O} 2}^{0}$ in $\beta-\mathrm{Ga}_{2} \mathrm{O}_{3}$ and $\mathrm{N}_{\mathrm{O} 6}^{0}$ in $\varepsilon-\mathrm{Ga}_{2} \mathrm{O}_{3}$, respectively. Unlike cation-site acceptor $\mathrm{Mg}_{\mathrm{Ga}}^{0}, \mathrm{~N}_{\mathrm{O}}$ traps a hole generated by itself with an $\mathrm{N} 2 p$-like state. Examining Fig. 5 reveals that the relative position of the $+/ 0$ thermodynamic transition level for the STH is related to the 0/acceptor level for $\mathrm{N}_{\mathrm{O}}$ on each site. As mentioned above, STHs cannot be stabilized on the $\mathrm{O} 2$ site in $\beta-\mathrm{Ga}_{2} \mathrm{O}_{3}$ or the $\mathrm{O} 2$, $\mathrm{O} 4$, and $\mathrm{O} 6$ sites in $\varepsilon-\mathrm{Ga}_{2} \mathrm{O}_{3}$. However, for $\mathrm{N}_{\mathrm{O}}$, the released holes are trapped by themselves on these sites [Fig. 6(d)], with acceptor levels lower than those on the other stable STH sites except for $\mathrm{N}_{\mathrm{O} 6}$ in $\varepsilon-\mathrm{Ga}_{2} \mathrm{O}_{3}$ [Figs. 5(b), 5(d), and 6(e)]. Furthermore, it seems that in the favorable sites for STHs, such as $\mathrm{O} 1$ and $\mathrm{O} 3$ in $\beta-\mathrm{Ga}_{2} \mathrm{O}_{3}$ and $\mathrm{O} 1, \mathrm{O} 3$, and $\mathrm{O} 5$ in $\varepsilon-\mathrm{Ga}_{2} \mathrm{O}_{3}$, the relative positions of the STH thermodynamic transition levels are related to those for the corresponding $\mathrm{N}_{\mathrm{O}}$.

We checked the gKT condition for $\mathrm{Mg}_{\mathrm{Ga}}^{0}$ and $\mathrm{N}_{\mathrm{O}}^{0}$ in $\beta-\mathrm{Ga}_{2} \mathrm{O}_{3}$ by adding an electron to the unoccupied acceptor levels $\varepsilon_{\mathrm{A}}(N)$. We find that $\operatorname{HSE}(0.35,0.21)$, which is used to reproduce the experimental band gap, well satisfies the gKT for $\mathrm{N}_{\mathrm{O} 3}$ with $\varepsilon_{\mathrm{A}}(N+1)-\varepsilon_{\mathrm{A}}(N)=0.03 \mathrm{eV}$. In contrast, $\operatorname{HSE}(0.35,0.21)$ does not satisfy the gKT well for $\mathrm{Mg}_{\mathrm{Ga} 1}$, $\mathrm{Mg}_{\mathrm{Ga} 2}, \mathrm{~N}_{\mathrm{O} 1}$, or $\mathrm{N}_{\mathrm{O} 2}$, being convex with $\varepsilon_{\mathrm{A}}(N+1)-\varepsilon_{\mathrm{A}}(N)=$ $0.24,0.34,0.13$, and $0.13 \mathrm{eV}$, respectively. Even if we adjust $\alpha$ and/or $\mu$ to satisfy the gKT condition, however, the positions of the acceptor levels are unlikely to change significantly because of the small $\alpha$ and $\mu$ dependence of $E(N)-E(N-1)$ found for the STH in Sec. III B. Therefore, we conclude that the $\mathrm{Mg}_{\mathrm{Ga}}$ and $\mathrm{N}_{\mathrm{O}}$ impurities are deep acceptors in all four $\mathrm{Ga}_{2} \mathrm{O}_{3}$ polymorphs, which is further evidence of the difficulty of their $p$-type doping.

\section{CONCLUSIONS}

We have investigated the stability of STHs and the acceptor levels of $\mathrm{Mg}$ and $\mathrm{N}$ impurities using the HSE hybrid functional to assess the $p$-type dopability of $\alpha-, \beta-, \delta$-, and $\varepsilon-\mathrm{Ga}_{2} \mathrm{O}_{3}$. Their electronic band structures have been obtained using $\operatorname{HSE}(0.35,0.21)$, which well reproduces the experimental gap of $\beta-\mathrm{Ga}_{2} \mathrm{O}_{3}$. All the polymorphs show valence and conduction bands with small and large energy dispersions, respectively. The electron effective mass is almost invariant with polymorph type, with values between $0.26 m_{0}$ and $0.28 m_{0}$.

The gKT condition of the HSE functional is discussed by taking a STH level in $\beta-\mathrm{Ga}_{2} \mathrm{O}_{3}$. The dependencies of the relevant quantities on the Fock-exchange mixing and screening parameters in HSE have been investigated including finite cell-size corrections for both total and single-particle energies. We found that the parameter values that well meet the gKT condition slightly overestimate the band gap, whlie $\operatorname{HSE}(0.35,0.21)$ shows slightly convex behavior. However, the absolute position of the STH level at a fixed geometry is nearly independent of $\alpha$ and $\mu$, showing that the results are robust against the choice of the parameters as long as the geometry and electronic structure of STHs are appropriately described and the band edges are well reproduced. Therefore, we have evaluated STH stability of the four $\mathrm{Ga}_{2} \mathrm{O}_{3}$ polymorphs using $\operatorname{HSE}(0.35,0.21)$. In all of the polymorphs, an introduced 
hole localizes on an $\mathrm{O}$ atom or two $\mathrm{O}$ atoms with atomic displacements. The resultant self-trapping energies are as high as $0.5 \mathrm{eV}$ or even higher in other cases. Furthermore, the acceptor levels for the substitutional $\mathrm{Mg}$ and $\mathrm{N}$ impurities have been calculated using $\operatorname{HSE}(0.35,0.21)$. Both $\mathrm{Mg}$ and $\mathrm{N}$ impurities introduce polaronic acceptor states, and their acceptor levels lie far above the VBM in all the polymorphs. In general, the acceptor levels are much higher than the STH levels, although the specific relationship between these levels depends on the sites for impurity-induced and self-trapped holes. Thus, $p$-type doping of the four $\mathrm{Ga}_{2} \mathrm{O}_{3}$ polymorphs seems unfeasible from the viewpoint of stable STH formation and the related deep, polaronic acceptor nature of the substitutional $\mathrm{Mg}$ and $\mathrm{N}$ impurities.

\section{ACKNOWLEDGMENTS}

This work was supported by CREST (Grant No. JPMJCR17J2) and the Support Program for Starting Up Innovation Hub MI ${ }^{2}$ I of the Japan Science and Technology Agency, and the Creation of Life Innovation Materials for Interdisciplinary and International Researcher Development Project of the Ministry of Education, Culture, Sports, Science and Technology of Japan. Computing resources of the Academic Center for Computing and Media Studies at Kyoto University were used.

\section{APPENDIX}

Here, we discuss the dependence of the gKT-relevant quantities and the self-trapping energies on the supercell size, taking an example of the STH in $\alpha-\mathrm{Ga}_{2} \mathrm{O}_{3}$ primitive-based supercells because it has a relatively simple crystal structure among the four polymorphs. The calculations were performed using PBE-GGA $+U$ [99] on the basis of Dudarev's formalism [100] with an effective $U$ value of $10 \mathrm{eV}$ applied to the $\mathrm{O} 2 p$ orbitals, which enables the stabilization of the STH and the use of large supercells. Figure 7(a) shows the corrected gKTrelevant quantities as a function of the supercell size. The STH single-particle level $\varepsilon_{\mathrm{ST}}(N-1)$ shifts downward by $\sim 0.2 \mathrm{eV}$ by electron addition $\left[\varepsilon_{\mathrm{ST}}(N)\right]$ for any cell size, indicating this approximation shows slight concave behavior. A notable message from Fig. 7(a) is that $\varepsilon_{\mathrm{ST}}(N)-\varepsilon_{\mathrm{ST}}(N-1)$ has already converged well with the smallest 80 -atom supercell. Moreover, $E(N)-E(N-1)$ is close in value to $\varepsilon_{\mathrm{ST}}(N-1)$ for any cell size. In other words, the differences between $E(N)-E(N-1)$ and $\varepsilon_{\mathrm{ST}}(N-1)$ or $\varepsilon_{\mathrm{ST}}(N)$ have also converged well. When the curve of total energy versus fractional electron number is quadratic, $\varepsilon_{\mathrm{ST}}(N-1)$ and $\varepsilon_{\mathrm{ST}}(N)$ are distributed symmetrically about $E(N)-E(N-1)$, as shown in Fig. 3. The $U$ correction enhances the concavity by adding a quadratic penalty function; however, the resultant curves are not quadratic and the derivative values at the integer $N-1$ are nearly on the straight line.

The self-trapping energies and the STH single-particle levels with and without corrections are shown in Fig. 7(b) as a function of the supercell size. Both corrected $E_{\mathrm{ST}}$ and
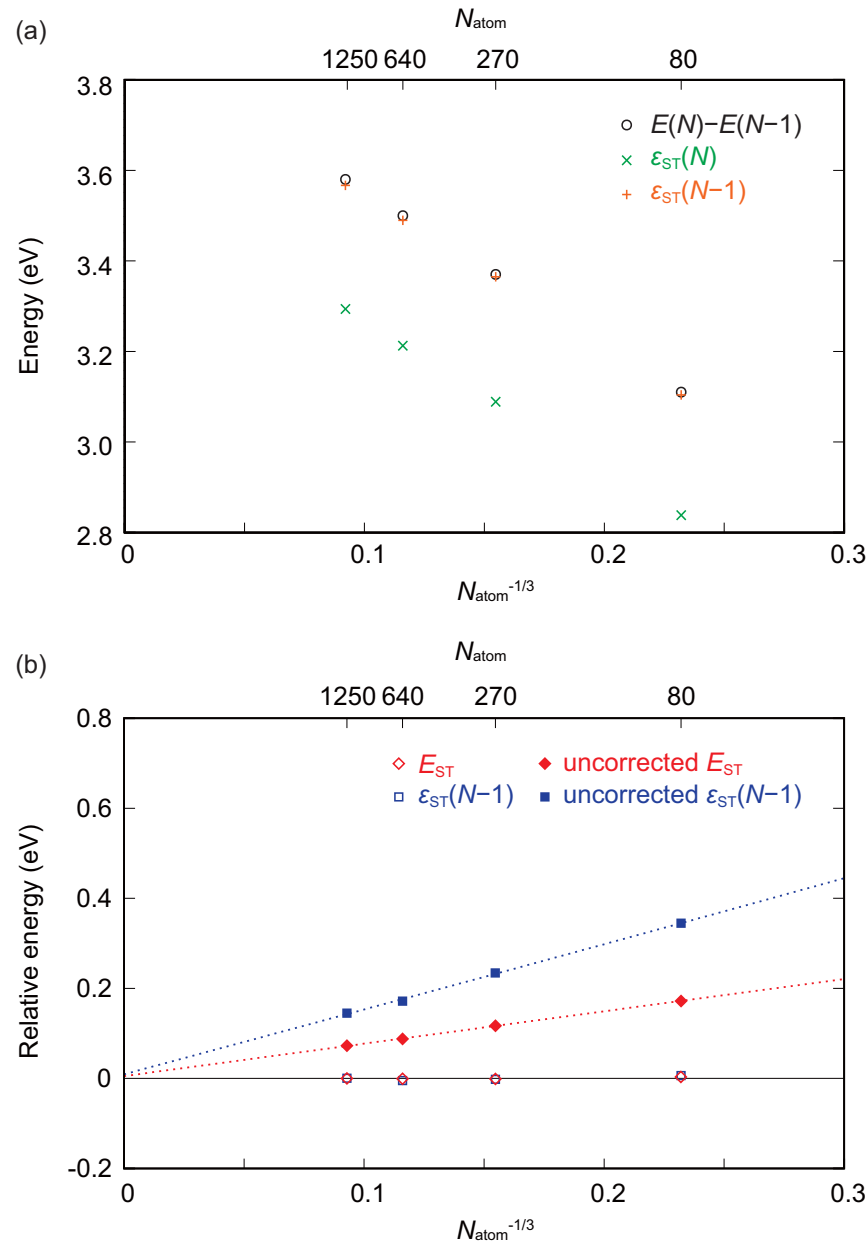

FIG. 7. Dependence of (a) corrected gKT-relavent quantities and (b) corrected or uncorrected self-trapping energies and STH singleparticle levels on supercell size for the STH in $\alpha-\mathrm{Ga}_{2} \mathrm{O}_{3} . N_{\text {atom }}$ is the number of atoms in the supercell. $\Gamma$-centered $k$-point meshes of $4 \times 4 \times 4$ and $2 \times 2 \times 2$ are used for the 80- and 270-atom supercells, respectively, while 640- and 1250-atom supercells are with the $\Gamma$-only sampling. The STHs are modeled using PBE$\mathrm{GGA}+U$, where an effective $U$ value of $10 \mathrm{eV}$ is applied to the $\mathrm{O}$ $2 p$ orbitals. The uncorrected self-trapping energies and STH singleparticle levels are fitted with a function of $a N_{\text {atom }}^{-1}+b N_{\text {atom }}^{-1 / 3}+c$ in (b); the energy zeros are set at the respective corrected values for the largest 1250-atom supercell.

$\varepsilon_{\mathrm{ST}}(N-1)$ converge rapidly at the smallest 80 -atom supercell. The nearly linear dependence indicates that the first-order term associated with point-charge contributions has a predominant influence on the cell-size dependence of $E_{\mathrm{ST}}$ and $\varepsilon_{\mathrm{ST}}(N-1)$ for the STH. The bottom line is that the gKTrelevant quantities, self-trapping energies, and STH singleparticle levels are all reproducible by relatively small supercells after the cell-size corrections using the schemes described in Sec. II C.
[1] N. Ueda, H. Hosono, R. Waseda, and H. Kawazoe, Appl. Phys. Lett. 70, 3561 (1997).
[2] M. Orita, H. Ohta, M. Hirano, and H. Hosono, Appl. Phys. Lett. 77, 4166 (2000). 
[3] T. Oshima, T. Okuno, N. Arai, N. Suzuki, S. Ohira, and S. Fujita, Appl. Phys. Express 1, 011202 (2008).

[4] M. Higashiwaki, K. Sasaki, A. Kuramata, T. Masui, and S. Yamakoshi, Appl. Phys. Lett. 100, 013504 (2012).

[5] D. Y. Guo, Z. P. Wu, Y. H. An, X. C. Guo, X. L. Chu, C. L. Sun, L. H. Li, P. G. Li, and W. H. Tang, Appl. Phys. Lett. 105, 023507 (2014).

[6] T. Oishi, Y. Koga, K. Harada, and M. Kasu, Appl. Phys. Express 8, 031101 (2015).

[7] M. A. Mastro, A. Kuramata, J. Calkins, J. Kim, F. Ren, and S. J. Pearton, ECS J. Solid State Sci. Technol. 6, P356 (2017).

[8] S. J. Pearton, J. Yang, P. H. Cary, F. Ren, J. Kim, M. J. Tadjer, and M. A. Mastro, Appl. Phys. Rev. 5, 011301 (2018).

[9] N. Suzuki, S. Ohira, M. Tanaka, T. Sugawara, K. Nakajima, and T. Shishido, Phys. Status Solidi (C) 4, 2310 (2007).

[10] E. G. Víllora, K. Shimamura, Y. Yoshikawa, T. Ujiie, and K. Aoki, Appl. Phys. Lett. 92, 202120 (2008).

[11] K. Iwaya, R. Shimizu, H. Aida, T. Hashizume, and T. Hitosugi, Appl. Phys. Lett. 98, 142116 (2011).

[12] J. B. Varley, J. R. Weber, A. Janotti, and C. G. Van de Walle, Appl. Phys. Lett. 97, 142106 (2010).

[13] H. Peelaers and C. G. Van de Walle, Phys. Rev. B 94, 195203 (2016).

[14] S. Lany, APL Mater. 6, 046103 (2018).

[15] A. Kyrtsos, M. Matsubara, and E. Bellotti, Appl. Phys. Lett. 112, 032108 (2018).

[16] J. L. Lyons, Semicond. Sci. Technol. 33, 05LT02 (2018).

[17] J. B. Varley, A. Janotti, C. Franchini, and C. G. Van de Walle, Phys. Rev. B 85, 081109 (2012).

[18] P. Deák, Q. Duy Ho, F. Seemann, B. Aradi, M. Lorke, and T. Frauenheim, Phys. Rev. B 95, 075208 (2017).

[19] Q. D. Ho, T. Frauenheim, and P. Deák, Phys. Rev. B 97, 115163 (2018).

[20] H. Kawazoe, M. Yasukawa, H. Hyodo, M. Kurita, H. Yanagi, and H. Hosono, Nature 389, 939 (1997).

[21] A. Togo, F. Oba, I. Tanaka, and K. Tatsumi, Phys. Rev. B 74, 195128 (2006).

[22] Y. Ogo, H. Hiramatsu, K. Nomura, H. Yanagi, T. Kamiya, M. Hirano, and H. Hosono, Appl. Phys. Lett. 93, 032113 (2008).

[23] H. Hosono, Transparent Oxide Semiconductors: Fundamentals and Recent Progress (John Wiley \& Sons, Ltd, UK, 2010), Chap. 2, pp. 31-59.

[24] J. B. Varley, A. Miglio, V.-A. Ha, M. J. van Setten, G.-M. Rignanese, and G. Hautier, Chem. Mater. 29, 2568 (2017).

[25] F. Oba and Y. Kumagai, Appl. Phys. Express 11, 060101 (2018).

[26] K. Yim, Y. Youn, M. Lee, D. Yoo, J. Lee, S. H. Cho, and S. Han, npj Comput. Mater. 4, 17 (2018).

[27] S. Lany and A. Zunger, Phys. Rev. B 80, 085202 (2009).

[28] S. Lany and A. Zunger, Appl. Phys. Lett. 96, 142114 (2010).

[29] J. L. Lyons, A. Janotti, and C. G. Van de Walle, J. Appl. Phys. 115, 012014 (2014).

[30] L. Weston, D. Wickramaratne, and C. G. Van de Walle, Phys. Rev. B 96, 100102 (2017).

[31] R. Roy, V. G. Hill, and E. F. Osborn, J. Am. Chem. Soc. 74, 719 (1952).

[32] S. Yoshioka, H. Hayashi, A. Kuwabara, F. Oba, K. Matsunaga, and I. Tanaka, J. Phys.: Condens. Matter 19, 346211 (2007).

[33] D. Shinohara and S. Fujita, Jpn. J. Appl. Phys. 47, 7311 (2008).
[34] D. Guo, X. Zhao, Y. Zhi, W. Cui, Y. Huang, Y. An, P. Li, Z. Wu, and W. Tang, Mater. Lett. 164, 364 (2016).

[35] A. Segura, L. Artús, R. Cuscó, R. Goldhahn, and M. Feneberg, Phys. Rev. Mater. 1, 024604 (2017).

[36] H. Ito, K. Kaneko, and S. Fujita, Jpn. J. Appl. Phys. 51, 100207 (2012).

[37] T. Wang, W. Li, C. Ni, and A. Janotti, Phys. Rev. Appl. 10, 011003 (2018).

[38] E. Chikoidze, H. J. von Bardeleben, K. Akaiwa, E. Shigematsu, K. Kaneko, S. Fujita, and Y. Dumont, J. Appl. Phys. 120, 025109 (2016).

[39] S. B. Cho and R. Mishra, Appl. Phys. Lett. 112, 162101 (2018).

[40] S. Kokott, Ph.D. thesis, Humboldt-Universität zu Berlin (2018).

[41] H. Hayashi, R. Huang, H. Ikeno, F. Oba, S. Yoshioka, I. Tanaka, and S. Sonoda, Appl. Phys. Lett. 89, 181903 (2006).

[42] T. Oshima, K. Matsuyama, K. Yoshimatsu, and A. Ohtomo, J. Cryst. Growth 421, 23 (2015).

[43] C. G. Van de Walle and J. Neugebauer, J. Appl. Phys. 95, 3851 (2004).

[44] A. M. Stoneham, J. Gavartin, A. L. Shluger, A. V. Kimmel, D. M. Ramo, H. M. Rønnow, G. Aeppli, and C. Renner, J. Phys.: Condens. Matter 19, 255208 (2007).

[45] B. J. Morgan, D. O. Scanlon, and G. W. Watson, J. Mater. Chem. 19, 5175 (2009).

[46] F. Oba, M. Choi, A. Togo, and I. Tanaka, Sci. Technol. Adv. Mater. 12, 034302 (2011).

[47] K. P. McKenna, M. J. Wolf, A. L. Shluger, S. Lany, and A. Zunger, Phys. Rev. Lett. 108, 116403 (2012).

[48] C. Freysoldt, B. Grabowski, T. Hickel, J. Neugebauer, G. Kresse, A. Janotti, and C. G. Van de Walle, Rev. Mod. Phys. 86, 253 (2014).

[49] P. Erhart, A. Klein, D. Åberg, and B. Sadigh, Phys. Rev. B 90, 035204 (2014).

[50] Y. Hinuma, T. Hatakeyama, Y. Kumagai, L. A. Burton, H. Sato, Y. Muraba, S. Iimura, H. Hiramatsu, I. Tanaka, H. Hosono, and F. Oba, Nat. Commun. 7, 11962 (2016).

[51] K. Matsuzaki, K. Harada, Y. Kumagai, S. Koshiya, K. Kimoto, S. Ueda, M. Sasase, A. Maeda, T. Susaki, M. Kitano, F. Oba, and H. Hosono, Adv. Mater. 30, 1801968 (2018).

[52] J. P. Perdew, R. G. Parr, M. Levy, and J. L. Balduz, Phys. Rev. Lett. 49, 1691 (1982).

[53] A. J. Cohen, P. Mori-Sánchez, and W. Yang, Science 321, 792 (2008).

[54] P. Mori-Sánchez, A. J. Cohen, and W. Yang, Phys. Rev. Lett. 100, 146401 (2008).

[55] A. V. Krukau, O. A. Vydrov, A. F. Izmaylov, and G. E. Scuseria, J. Chem. Phys. 125, 224106 (2006).

[56] J. Paier, M. Marsman, K. Hummer, G. Kresse, I. C. Gerber, and J. G. Ángyán, J. Chem. Phys. 124, 154709 (2006).

[57] E. R. Batista, J. Heyd, R. G. Hennig, B. P. Uberuaga, R. L. Martin, G. E. Scuseria, C. J. Umrigar, and J. W. Wilkins, Phys. Rev. B 74, 121102 (2006).

[58] A. Alkauskas, P. Broqvist, and A. Pasquarello, Phys. Status Solidi (B) 248, 775 (2011).

[59] Y. Kumagai, M. Choi, Y. Nose, and F. Oba, Phys. Rev. B 90, 125202 (2014).

[60] Y. Hinuma, A. Grüneis, G. Kresse, and F. Oba, Phys. Rev. B 90, 155405 (2014). 
[61] A. Grüneis, G. Kresse, Y. Hinuma, and F. Oba, Phys. Rev. Lett. 112, 096401 (2014).

[62] J. Heyd, G. E. Scuseria, and M. Ernzerhof, J. Chem. Phys. 118, 8207 (2003).

[63] M. Marsman, J. Paier, A. Stroppa, and G. Kresse, J. Phys.: Condens. Matter 20, 064201 (2008).

[64] F. Oba, A. Togo, I. Tanaka, J. Paier, and G. Kresse, Phys. Rev. B 77, 245202 (2008).

[65] S. Lany and A. Zunger, Phys. Rev. B 81, 205209 (2010).

[66] T. Stein, H. Eisenberg, L. Kronik, and R. Baer, Phys. Rev. Lett. 105, 266802 (2010).

[67] G. Miceli, W. Chen, I. Reshetnyak, and A. Pasquarello, Phys. Rev. B 97, 121112 (2018).

[68] C. Freysoldt, J. Neugebauer, and C. G. Van de Walle, Phys. Rev. Lett. 102, 016402 (2009).

[69] Y. Kumagai and F. Oba, Phys. Rev. B 89, 195205 (2014).

[70] P. E. Blöchl, Phys. Rev. B 50, 17953 (1994).

[71] G. Kresse and J. Furthmüller, Phys. Rev. B 54, 11169 (1996).

[72] G. Kresse and D. Joubert, Phys. Rev. B 59, 1758 (1999).

[73] S. Lany and A. Zunger, Phys. Rev. B 81, 113201 (2010).

[74] M. Jain, J. R. Chelikowsky, and S. G. Louie, Phys. Rev. Lett. 107, 216803 (2011).

[75] H.-P. Komsa, T. T. Rantala, and A. Pasquarello, Phys. Rev. B 86, 045112 (2012).

[76] W. Chen and A. Pasquarello, Phys. Rev. B 88, 115104 (2013).

[77] J. P. Perdew, K. Burke, and M. Ernzerhof, Phys. Rev. Lett. 77, 3865 (1996).

[78] S. Baroni and R. Resta, Phys. Rev. B 33, 7017 (1986).

[79] M. Gajdoš, K. Hummer, G. Kresse, J. Furthmüller, and F. Bechstedt, Phys. Rev. B 73, 045112 (2006).

[80] R. W. Nunes and X. Gonze, Phys. Rev. B 63, 155107 (2001).

[81] H. He, R. Orlando, M. A. Blanco, R. Pandey, E. Amzallag, I. Baraille, and M. Rérat, Phys. Rev. B 74, 195123 (2006).

[82] H. Peelaers and C. G. Van de Walle, Phys. Status Solidi (B) 252, 828 (2015).
[83] J. Furthmüller and F. Bechstedt, Phys. Rev. B 93, 115204 (2016)

[84] Y. Hinuma, G. Pizzi, Y. Kumagai, F. Oba, and I. Tanaka, Comput. Mater. Sci. 128, 140 (2017).

[85] G. Sinha, K. Adhikary, and S. Chaudhuri, J. Cryst. Growth 276, 204 (2005).

[86] M. Mohamed, C. Janowitz, I. Unger, R. Manzke, Z. Galazka, R. Uecker, R. Fornari, J. R. Weber, J. B. Varley, and C. G. Van de Walle, Appl. Phys. Lett. 97, 211903 (2010).

[87] J. P. Perdew, M. Ernzerhof, and K. Burke, J. Chem. Phys. 105, 9982 (1996)

[88] D. Cahen and A. Kahn, Adv. Mater. 15, 271 (2003).

[89] Y. Hinuma, F. Oba, Y. Kumagai, and I. Tanaka, Phys. Rev. B 86, 245433 (2012).

[90] Y. Kumagai, L. A. Burton, A. Walsh, and F. Oba, Phys. Rev. Appl. 6, 014009 (2016).

[91] Y. Kumagai, K. T. Butler, A. Walsh, and F. Oba, Phys. Rev. B 95, 125309 (2017).

[92] Y. Hinuma, Y. Kumagai, I. Tanaka, and F. Oba, Phys. Rev. Mater. 2, 124603 (2018).

[93] M. Mohamed, K. Irmscher, C. Janowitz, Z. Galazka, R. Manzke, and R. Fornari, Appl. Phys. Lett. 101, 132106 (2012).

[94] D. West, Y. Y. Sun, and S. B. Zhang, Appl. Phys. Lett. 101, 082105 (2012).

[95] Y. Hinuma, Y. Kumagai, I. Tanaka, and F. Oba, Phys. Rev. B 95, 075302 (2017).

[96] Y. Hinuma, Y. Kumagai, F. Oba, and I. Tanaka, Comput. Mater. Sci. 113, 221 (2016).

[97] J. P. Perdew, A. Ruzsinszky, G. I. Csonka, O. A. Vydrov, G. E. Scuseria, L. A. Constantin, X. Zhou, and K. Burke, Phys. Rev. Lett. 100, 136406 (2008).

[98] Y. Hinuma, H. Hayashi, Y. Kumagai, I. Tanaka, and F. Oba, Phys. Rev. B 96, 094102 (2017).

[99] V. I. Anisimov, J. Zaanen, and O. K. Andersen, Phys. Rev. B 44, 943 (1991).

[100] S. L. Dudarev, G. A. Botton, S. Y. Savrasov, C. J. Humphreys, and A. P. Sutton, Phys. Rev. B 57, 1505 (1998). 